\title{
Sumsets avoiding squarefree integers
}

by

\section{Jan-Christoph Schlage-Puchta (Gent)}

1. Introduction and results. Let $A \subseteq[1, x] \cap \mathbb{N}$ be a set of $\delta x$ integers. Erdős [1] asked whether $\delta>1 / 4$ implies that there is some subset of $A$ adding up to a squarefree integer. Erdős and Freiman [2] showed that this is indeed the case; in fact, $\mathcal{O}(\log x)$ summands are already sufficient. This was further improved by Nathanson and Sárközy [4, who showed that 21 summands suffice, and Filaseta [3] showed that if $\delta>1-8 / \pi^{2}+\epsilon$ and $x>x_{0}(\epsilon)$, then either $A+A$ contains a squarefree integer, or $A$ is a subset of $4 \mathbb{N}$ or a subset of $4 \mathbb{N}+2$. Schoen [5] showed that if $\delta \geq 0.1$ and $x$ is sufficiently large, then either $A+A$ contains a squarefree integer, or $A$ is a subset of $4 \mathbb{N}, 9 \mathbb{N}$, or $4 \mathbb{N}+2$. From this sequence of results one might expect that for every $\delta>0$ a set $A$ with $|A|>\delta x$ such that $A+A$ contains no squarefree integer does so because $A+A$ is not much larger than $A$ itself. However, here we show that this is only the case for $\delta$ not too small. More precisely, we show that the values $\delta=1 / 4-2 / \pi^{2}=0.0473 \ldots$ and $\delta_{1}=1 / 18$ are critical. We will show the following.

Theorem 1. Set $\delta_{0}=1 / 4-2 / \pi^{2}$ and $\delta_{1}=1 / 18$. For every $\epsilon>0$ there exists some $x_{0}$ such that for all $x>x_{0}$ the following statements hold.

(i) Let $A \subseteq[1, x] \cap \mathbb{N}$ be a set of integers such that $A+A$ contains no squarefree integer. If $|A|>\left(\delta_{1}+\epsilon\right) x$, then $A$ is a subset of $4 \mathbb{N}, 9 \mathbb{N}$, or $4 \mathbb{N}+2$.

(ii) Let $A \subseteq[1, x] \cap \mathbb{N}$ be a set of integers such that $A+A$ contains no squarefree integers. If $|A|>\left(\delta_{0}+\epsilon\right) x$, then either $A$ is as in (i), or all elements of $A$ are even, and there exists some $a \in\{1, \ldots, 8\}$ such that for $n \in A$ we have

$$
\begin{aligned}
& n \equiv 2(\bmod 4) \Leftrightarrow n \equiv a(\bmod 9), \\
& n \equiv 0(\bmod 4) \Leftrightarrow n \equiv-a(\bmod 9) .
\end{aligned}
$$

2010 Mathematics Subject Classification: Primary 11P70; Secondary 11N25, 11B75.

Key words and phrases: squarefree integers, sumsets, inverse problems. 
(iii) There exists a set $A \subseteq \mathbb{N}$ of density $>\delta_{0}-\epsilon$ such that $A+A$ contains no squarefree integers, $A+A+A$ contains all integers with finitely many exceptions, and the set of non-squarefree integers not contained in $A+A$ has density at most $\epsilon$.

A more qualitative description for infinite sets is given in the following. For a set $A$, let $\Sigma(A)$ be the set of all subset sums of $A$.

Corollary 2. Let $A \subseteq \mathbb{N}$ be a set of upper density $\delta$, and assume that $A+A$ contains no squarefree integers. If $\delta>1 / 18$, then $\Sigma(A+A)$ has density at most $1 / 4$. If $\delta>1 / 4-2 / \pi^{2}$, then $\Sigma(A)$ has density at most $1 / 2$. On the other hand, there exists a set $A$ of density $1 / 18$ such that $A+A$ contains no squarefree integer, but $A+A+A$ does, and every sufficiently large even integer can be written as the sum of six elements of $A$. Moreover, for every $\epsilon>0$ there exists a set $A$ of density $1 / 4-2 / \pi^{2}-\epsilon$ such that $A+A$ contains no squarefree integer, $A+A$ has density $1-6 / \pi^{2}-\epsilon$, and $A+A+A=\mathbb{N}$ with finitely many exceptions.

We remark that Schoen [5] constructed a set $A$ of density $\frac{1}{36}+0.00025 \ldots$ such that $A+A$ contains no squarefree integer, but for any periodic set $B \supseteq A, B+B$ contains squarefree integers.

2. Proof of parts (i) and (ii). Let $A \subset[1, x] \cap \mathbb{N}$ be a set such that $A>\left(\delta_{0}+\epsilon\right) x$ and $A+A$ contains no squarefree integer. Assume further that $A$ is not a subset of $4 \mathbb{N}, 9 \mathbb{N}$, or $4 \mathbb{N}+2$. The proof relies on a detailed study of the distribution of $A$ modulo 36. For each $a \in\{0, \ldots, 35\}$ set

$$
\delta_{a}=\frac{36 \#\{n \in A: n \equiv a(\bmod 36)\}}{x},
$$

that is, $\delta_{a}$ is the local density of $A$ in the residue class $a \bmod 36$. Let

$$
\begin{aligned}
& U=\left\{a \in\{0, \ldots, 35\}: \delta_{a}>1-9 / \pi^{2}+\epsilon / 100\right\}, \\
& V=\left\{a \in\{0, \ldots, 35\}: \delta_{a}>0\right\} .
\end{aligned}
$$

Finally, let

$$
Q=\{0,4,8,9,12,16,18,20,24,27,28,32\}
$$

be the set of all residue classes mod 36 which do not contain squarefree integers.

Lemma 3. We have $U+V \subseteq Q$.

Proof. Suppose that $u \in U, v \in V$, and $a+b \notin Q$. Fix $b \in A$ with $b \equiv v(\bmod 36)$. Consider the set $\{a+b: a \in A, a \equiv u(\bmod 36)\}$. None of its elements is squarefree. However, the density of squarefree integers in a residue class modulo 36 which is not in $Q$ is

$$
\frac{4}{3} \cdot \frac{9}{8} \cdot \zeta(2)^{-1}=\frac{9}{\pi^{2}}
$$


Since $\delta_{a}>1-9 / \pi^{2}+\epsilon / 100$, and $x$ is sufficiently large, this yields a contradiction.

Lemma 4. If $|U| \geq 2$, we are in the situation described in Theorem 1(ii).

Proof. Suppose first that $U$ contains three different elements $a, b, c$. Assume first that $a$ is odd. Then $2 a \equiv 18(\bmod 36)$, thus $a$ is 9 or 27 . In particular, one of $b$ and $c$ is even, say, $b$ is even. Then $a+b$ is odd, hence $b$ is 0 or 18. No matter whether $c$ is odd or even, it is always divisible by 9 . If every element in $V$ is divisible by 9 , then $A \subseteq 9 \mathbb{N}$, and we are done. Otherwise there exists $v \in V$ which is not divisible by 9 . Then $a+v, b+v, c+v \in Q$. Since $a$ is odd, this implies that $v$ is odd; but then $b+v$ is also odd, hence divisible by 9 , which is impossible since $b$ is divisible by 9 , but $v$ is not. Hence, if $|U| \geq 3$, then all elements in $U$ are even. Moreover, if some $v \in V$ is odd, then $U+v$ contains at least three odd elements, which is impossible, since $Q$ contains only two odd elements. Hence $A \subseteq 2 \mathbb{N}$. If there are $u \in U$ and $v \in V$ with $u \not \equiv v(\bmod 4)$, then we have both $u+v \in Q$ and $u+v \equiv 2$ $(\bmod 4)$, which implies $u+v=18$. Hence, for each $v \in V$ there is at most one $u \in U$ with $v \not \equiv u(\bmod 4)$. However, since $|V| \geq|U| \geq 3$, this is impossible, and we conclude that all elements in $V$ are congruent modulo 4 . As this case was excluded at the beginning, we find that $|U| \geq 3$ is impossible.

Now consider the case $|U|=2$, and assume first that $|V| \geq 3$. If one element of $U$ is odd, it has to be 9 or 27 , and $V$ does not contain any even element except possibly 0 and 18. If $U$ contains another odd element, then $U=\{9,27\}$. Then $V \backslash U$ contains at least one odd element $a$, for otherwise we would have $V \subseteq\{0,9,18,27\}$, which is impossible since $A \nsubseteq 9 \mathbb{N}$. For this $a$ both $9+a, 27+a$ are divisible by 4 , which is impossible. Hence, $U$ contains one odd and one even element; more precisely, $U=\left\{u_{1}, u_{2}\right\}$, where $u_{1} \in\{9,27\}$ and $u_{2} \in\{0,18\}$. Let $v \in V \backslash U$ be an element which is not divisible by 9 . If no such element exists, then $A \subseteq 9 \mathbb{N}$, and we are done. If such an element exists, neither $v+u_{1}$ nor $v+u_{2}$ is divisible by 9 , and at least one of them is odd, that is, one of $v+u_{1}, v+u_{2}$ is not in $Q$. Hence, all elements in $U$ are even.

If an element of $V$ is odd, the elements in $U$ have difference 18, thus their sum is not divisible by 4 , and we conclude that $U=\{0,18\}$. Hence, all elements in $V \backslash U$ are odd, which implies that $V \backslash U \subseteq\{9,27\}$, contradicting the assumption that $A$ is not contained in $9 \mathbb{N}$. Hence, all elements in $A$ are even. If they are not all congruent modulo 4 , then there are at least two sums $u+v$ which are 2 modulo 4 , which is impossible. Hence, $|U|=2$ and $|V| \geq 3$ is also impossible.

Thus, it remains to consider the case $U=V=\left\{u_{1}, u_{2}\right\}$. If $u_{1}$ is odd, then $2 u_{1}=18$, that is, $u_{1}$ is 9 or 27 . If $u_{2}$ were also odd, then we would have $U=\{9,27\}$, that is, $A \subseteq 9 \mathbb{N}$. If $u_{2}$ were even, the only possibility is 
$u_{2}=18$, which gives the same contradiction. Hence, both $u_{1}, u_{2}$ are even. If they are congruent to each other modulo 4 , we would obtain $A \subseteq 4 \mathbb{N}$ or $A \subseteq 4 N+2$. Hence, their sum is an element in $Q$ which is $2(\bmod 4)$, hence $u_{1}+u_{2} \equiv 18(\bmod 36)$. But this implies the description in part (ii).

Lemma 5. The case $U=\emptyset$ is impossible.

Proof. We have

$$
36 \delta_{0}-12\left(1-9 / \pi^{2}\right) \geq 24 \cdot 0.0269,
$$

hence for every $a \in V$ there exists some $b$ such that $\delta_{b} \geq 0.0269$ and $a+b \notin Q$. In particular, we can find $b_{1}, b_{2} \in A$ with $\left|b_{1}-b_{2}\right| \leq 40$ and $b_{i} \equiv b(\bmod 36)$. As $x \rightarrow \infty$, the number of integers $n \equiv a(\bmod 36)$ such that both $n+b_{1}$ and $n+b_{2}$ are non-squarefree is asymptotically equal to

$$
\begin{aligned}
\frac{x}{36}\left(1-2 \prod_{p \geq 5}\right. & \left.\left(1-\frac{1}{p^{2}}\right)+\prod_{\substack{p \geq 5 \\
p^{2} \mid b_{1}-b_{2}}}\left(1-\frac{1}{p^{2}}\right) \prod_{\substack{p \geq 5 \\
p^{2} \nmid b_{1}-b_{2}}}\left(1-\frac{2}{p^{2}}\right)\right) \\
& \leq \frac{x}{36}\left(1-\frac{18}{\pi^{2}}+\frac{24}{25} \prod_{p \geq 7}\left(1-\frac{2}{p^{2}}\right)\right)=0.04266 \ldots \cdot \frac{x}{36},
\end{aligned}
$$

hence for $x$ sufficiently large we obtain $\delta_{a} \leq 0.04267$ for all $a$. But this is impossible, since $\delta_{0}=0.0473>0.04267$.

Lemma 6. Let $a, b \in V$ with $a+b \notin Q$, and let $\epsilon>0$ be given. Then for $N$ sufficiently large we have

$$
\delta_{a}+\frac{3}{8} \delta_{b} \leq 1-\frac{9}{\pi^{2}}+\epsilon .
$$

Proof. Since $a, b \in V$ means that $\delta_{a}, \delta_{b}$ are positive, we see that none of $a, b$ is in $U$.

For the proof we will show that a lower bound for $\delta_{b}$ implies that $A$ contains elements $b_{1}, b_{2} \equiv b(\bmod 36)$ with difference not divisible by too many prime squares. This will then yield an upper bound for $\delta_{a}$, and comparing the bounds yields our claim.

Let $k \geq 3$ be the least integer such that $A$ contains $b_{1}, b_{2}$ with $p_{k}^{2} \nmid b_{1}-b_{2}$. Then $\delta_{b} \leq \prod_{i=3}^{k-1} p_{i}^{-2}+\epsilon$, and $\delta_{a}$ is at most $\epsilon$ plus the density of the integers $n \equiv a(\bmod 36)$ such that both $n+b_{1}$ and $n+b_{2}$ are non-squarefree. The density of the integers $n$ such that both $n+b_{1}$ and $n+b_{2}$ are squarefree equals

$$
\prod_{\substack{p \geq 5 \\ p^{2} \mid b_{1}-b_{2}}}\left(1-\frac{1}{p^{2}}\right) \prod_{\substack{p \geq 5 \\ p^{2} \nmid b_{1}-b_{2}}}\left(1-\frac{2}{p^{2}}\right) \leq\left(1-\frac{1}{p_{k}^{2}-1}\right) \frac{9}{\pi^{2}} .
$$


The density of the integers $n$ such that neither of $n+b_{1}, n+b_{2}$ is squarefree is therefore at most

$$
1-\frac{18}{\pi^{2}}+\left(1-\frac{1}{p_{k}^{2}-1}\right) \frac{9}{\pi^{2}}=1-\frac{9}{\pi^{2}}-\frac{9}{\pi^{2}\left(p_{k}^{2}-1\right)} .
$$

Hence,

$$
\delta_{a}+\frac{3}{8} \delta_{b} \leq 1-\frac{9}{\pi^{2}}-\frac{9}{\pi^{2}\left(p_{k}^{2}-1\right)}+\frac{3}{8} \prod_{i=3}^{k-1} p_{i}^{-2}+\epsilon \leq 1-\frac{9}{\pi^{2}}+\epsilon,
$$

provided that

$$
\frac{9}{\pi^{2}\left(p_{k}^{2}-1\right)} \geq \frac{3}{8} \prod_{i=3}^{k-1} p_{i}^{-2}
$$

which follows for $k \geq 5$ from $p_{k}<2 p_{k-1}$, and for $k=4$ by direct inspection. Hence, we find that $k=3$, and obtain $\delta_{a} \leq 1-9 / \pi^{2}-0.379$. Thus the bound $\delta_{a}+\frac{3}{8} \delta_{b} \leq 1-9 / \pi^{2}+\epsilon$ could only fail if $\delta_{b}>0.101$. But then there are $b_{1}, b_{2} \in A$ with $b_{1}, b_{2} \equiv b(\bmod 36)$ and $\left|b_{1}-b_{2}\right|<25$, thus $b_{1}-b_{2}$ is not divisible by the square of any prime different from 2,3 . This implies

$$
\delta_{a} \leq 1-\frac{18}{\pi^{2}}+\prod_{p \geq 5}\left(1-\frac{2}{p^{2}}\right)+\epsilon \leq 0.0066,
$$

and the relation $\delta_{a}+\frac{3}{8} \delta_{b} \leq 1-9 / \pi^{2}+\epsilon$ holds unless $\delta_{b}>0.217$, which is impossible since we already know that $b \notin U$.

Lemma 7. Suppose that $2 a \notin Q$. Then $\delta_{a} \leq 0.04+\epsilon$.

Proof. If $\delta_{a}>0.04+\epsilon$, then there exist $a_{1}, a_{2} \in A$ with $a_{1}, a_{2} \equiv a$ ( $\bmod 36)$ and $\left|a_{1}-a_{2}\right|<25$. Hence, $a_{1}-a_{2}$ is not divisible by the square of any prime different from 2 and 3 , and the same argument as in the previous lemma now implies $\delta_{a} \leq 0.0066$, which proves our claim.

Lemma 8. The case $|U|=1$ is impossible.

Proof. If $U=\{u\}$, then $A$ is concentrated in 12 residue classes modulo 36; more precisely, we have $V \subseteq Q-u$. On the other hand, we have

$$
\delta_{0}=\frac{1}{36}\left(1+8\left(1-9 / \pi^{2}\right)\right),
$$

and therefore $V \geq 9$. Assume first that $u$ is odd. Then at most two elements in $V$ are even, hence $V$ contains at most four elements $v$ with $2 v \in Q$. Thus, we have $\delta_{u} \leq 1, \delta_{a} \leq 1-9 / \pi^{2}+\epsilon$ for three more classes $a_{1}, a_{2}, a_{3} \in V$, and $\delta_{a} \leq 0.04+\epsilon$ for the remaining eight classes. Hence,

$$
|A| \leq \frac{x}{36}\left(1+3\left(1-9 / \pi^{2}\right)+8 \cdot 0.04+\epsilon\right) \leq 0.0441 x,
$$

which gives a contradiction since $\delta_{0}=0.0473 \ldots$ Now assume that $u$ is even. Every $v \in V$ satisfies precisely one of the two conditions $v+u \equiv 0$ 
$(\bmod 4)$ or $v+u \equiv 0(\bmod 9)$. Let $v_{1}, v_{2} \in V \backslash\{u\}$ be such that $v_{1}$ satisfies the first, and $v_{2}$ the second condition. Then

$$
v_{1}+v_{2} \equiv 4 x+9 y+2 u(\bmod 36),
$$

where $x=\frac{v_{1}-u}{4} \not \equiv 0(\bmod 9)$ and $y=\frac{v_{2}-u}{9} \not \equiv 0(\bmod 4)$. Since $u$ is even, the right hand side is not divisible by 4 . If it were divisible by 9 , then we obtain the relations $v_{1}+v_{2} \equiv u+v_{2} \equiv 0(\bmod 9)$, that is, $v_{1} \equiv u(\bmod 9)$. Together with $v_{1}+u \equiv 0(\bmod 4)$ and the fact that $u$ is even we obtain $v_{1} \equiv u(\bmod 36)$, a contradiction. Hence, if we set $V_{1}=\{v \in V: v \neq u$, $4 \mid v-u\}$, and $V_{2}=\{v \in V: v \neq u, 9 \mid v-u\}$, then $\left(V_{1}+V_{2}\right) \cap Q=\emptyset$. Set $\delta_{1}=\max _{v \in V_{1}} \delta_{v}$ and $\delta_{2}=\max _{v \in V_{2}} \delta_{v}$. Then from Lemma 6 we find that $\delta_{1}+\frac{3}{8} \delta_{2} \leq 1-9 / \pi^{2}+\epsilon$, hence

$$
\begin{aligned}
|A| & \leq \frac{x}{36}\left(1+8 \delta_{1}+3 \delta_{2}\right)=\frac{x}{36}\left(1+8\left(\delta_{1}+3 \frac{3}{8} \delta_{2}\right)\right) \\
& \leq \frac{x}{36}\left(1+8\left(1-9 / \pi^{2}\right)+\epsilon\right)=\left(\delta_{0}+\epsilon\right) x .
\end{aligned}
$$

Hence, our claim follows.

3. Proof of part (iii) and the corollary. Our proof is constructive. Denote by $p_{i}$ the $i$ th prime number. Fix an integer $k \geq 2$, and set $q=$ $\prod_{i=2}^{k} p_{i}^{2}$. Then we define

$$
A=\{n: 4 \mid n,(n, q) \text { squarefree }\} \cup\{n: q \mid n\}=A_{1} \cup A_{2},
$$

say. We claim that $A+A$ does not contain squarefree integers. In fact, every element of $A_{1}+A_{1}$ is divisible by 4 , while every element of $A_{2}+A_{2}$ is divisible by $q$, and $q$ is obviously a square. If $x \in A_{1}$ and $y \in A_{2}$, then $(x+y, q)=(x, q)$ is not squarefree, that is, $x+y$ has a divisor which is not squarefree, and is therefore itself not squarefree.

The density of $A_{1}$ is

$$
\frac{1}{4}\left(1-\prod_{i=2}^{k}\left(1-\frac{1}{p_{i}^{2}}\right)\right),
$$

which converges for $k \rightarrow \infty$ to

$$
\frac{1}{4}-\frac{1}{3} \zeta(2)^{-1}=\frac{1}{4}-\frac{2}{\pi^{2}}=\delta_{0} .
$$

Next, we show that $A+A+A$ contains all but finitely many integers. To do so note that since $k \geq 2$ we know that $A_{1}$ contains all multiples of 36 as well as all multiples of 100 . Hence, $A_{1}+A_{1}$ contains all integers divisible by 4 with finitely many exceptions, the largest of which is 764 . Now, $A_{2}$ contains all integers divisible by $q$, and since $q$ is odd, we see that $0, q, 2 q, 3 q$ are different residues modulo 4 , thus, every integer $>764+3 q$ can be written 
as the sum of a multiple of 36 , a multiple of 100 , and a multiple of $q$, thus $A_{1}+A_{1}+A_{2}$ contains all integers with finitely many exceptions.

Finally, we have to bound the number of non-squarefree integers which are not contained in $A+A$. Let $n$ be an integer divisible by $p^{2}$. If $p=2$ and $n \geq 764$, we can write $n=36 x+100 y$, thus $n \in A+A$. If $p=p_{i}, 2 \leq i \leq k$, and $n>4 q$, we can write $n$ as $4 p^{2} x+q y$, thus $n \in A+A$. Hence, the density of non-squarefree numbers not contained in $A+A$ is at most $\sum_{i>k} 1 / p_{i}^{2}$, which tends to 0 as $k$ goes to infinity.

We now prove the corollary. The only thing which is not obvious from the theorem are the claimed properties of the set constructed in Theorem 1(ii). We take $a=1$. Then every integer $n \equiv 30(\bmod 36)$ can be written as the sum of three elements of $A$, and this residue class contains infinitely many squarefree integers. To show that every sufficiently large even integer is the sum of six elements in $A$ it suffices to show that every sufficiently large integer which is divisible by 4 is the sum of five elements of $A$, that is, we have to show that every residue class $n$ modulo 9 can be written as $n=2 x-y$, where $x, y$ are natural numbers with $0<2 x+y \leq 5$. However, this can be checked without much effort.

\section{References}

[1] P. Erdös, Some problems and results on combinatorial number theory, in: Graph Theory and its Applications: East and West (Jinan, 1986), Ann. New York Acad. Sci. 576, New York Acad. Sci., New York, 1989, 132-145.

[2] P. Erdős and G. A. Freiman, On two additive problems, J. Number Theory 34 (1990), $1-12$.

[3] M. Filaseta, Sets with elements summing to squarefree numbers, C. R. Math. Rep. Acad. Sci. Canada 11 (1987), 243-246.

[4] M. B. Nathanson and A. Sárközy, Sumsets containing long arithmetic progressions and powers of 2, Acta Arith. 54 (1989), 147-154.

[5] T. Schoen, Squarefree numbers in sumsets, Indag. Math. (N.S.) 16 (2005), 251-265.

Jan-Christoph Schlage-Puchta

Building S22

Krijgslaan 281

9000 Gent, Belgium

E-mail: jcsp@cage.ugent.be 